\title{
The High Energy cosmic-Radiation Detection facility (HERD)
}

\author{
Fabio Gargano* on behalf of the HERD collaboration \\ INFN-Bari \\ E-mail: fabio.gargano@ba.infn.it
}

The High Energy cosmic-Radiation Detection (HERD) facility has been proposed as space astronomy payloads on-board the future China's Space Station (CSS) aimed to detect charged cosmicrays and gamma-rays from few $\mathrm{GeV}$ to $\mathrm{PeV}$ energies.

The main science objectives of HERD are searching dark matter particles, the study of cosmic ray chemical composition up to the knee and high energy gamma-ray observations. HERD consists of a very thick (3 Interaction Length, 55 Radiation Length) cube calorimeter made of small LYSO cubic crystals allowing 3D reconstruction, a tracker consisting of silicon microstrip detectors and thin converter foils for gamma conversion and plastic scintillator planes for veto and charge measurements. HERD will extend high precision and high statistics measurement of cosmicray spectra up to $1 \mathrm{PeV}$. It will also observe the gamma-ray sky from a few $\mathrm{GeV}$ up to $1 \mathrm{TeV}$ contributing to multi-messenger astronomy together with high energy gamma-ray, neutrino and gravitational waves ground-based telescopes.

European Physical Society Conference on High Energy Physics - EPS-HEP2019 -

10-17 July, 2019

Ghent, Belgium

${ }^{*}$ Speaker. 


\section{Introduction}

It is well established that neutral, cold/warm and non-baryonic dark matter (DM) dominates the total matter content in the universe. One way to detect DM is to search in space for its annihilation/decay products, which may lead to characteristic features in the observed spectra of cosmic electrons (plus positron) or gamma-ray spectra.

The steepening of the primary cosmic ray (CR) spectrum around PeV,the so-called "knee" region, is a classic but still unresolved problem in CR physics since its discovery in 1958. Space experiments, which can measure the particle energy and charge directly, have been suffering from small geometrical factors and limited energy range to make statistically meaningful measurements of the "knee".

The wide Filed Of View (FOV) space gamma-ray telescopes often provide crucial guidance to the observations of the ground-based narrow FOV telescopes. Unfortunately, the much more powerful ground-based Cherenkov Telescope Array (CTA) currently under development may not have the much-needed guidance from space wide FOV gamma-ray telescope, once the Fermi satellite stops operations. A new wide FOV space gamma-ray telescope is urgently needed to replace Fermi.

In order to address the above major problems in fundamental physics and astrophysics, the High Energy cosmic-Radiation Detection (HERD) facility has been proposed as one of several space astronomy payloads on-board the future China's Space Station (CSS), which is planned for operation starting around 2025 for about 10 years.

The primary scientific objectives of HERD are: (1) searching for signatures of the annihilation products of dark matter particles in the energy spectra and anisotropy of high energy electrons and gamma-rays from $500 \mathrm{MeV}$ to $100 \mathrm{TeV}$; (2) measuring precisely and directly the energy spectra and composition of primary cosmic rays from $10 \mathrm{GeV}$ up to PeV. The secondary scientific objectives of HERD include wide FOV monitoring of the high energy gamma-ray sky from $500 \mathrm{MeV}$ for gamma-ray bursts, active galactic nuclei and Galactic microquasars.

\section{Scientific Objectives}

\subsection{Measurement of comic electron spectrum and Dark Matter signal Search}

Numerous astrophysical and cosmological observations have shown that the normal baryonic matter only occupies $\sim 15 \%$ of the matter component in the universe, while the faction of dark matter (DM) is $\sim 85 \%$. The observations indicate that DM particles should be stable, neutral, and non-relativistic. However, the microscopic properties of DM particles remain unclear. Since the standard model of particle physics cannot provide a suitable DM candidate, the interpretation of DM requires new physics beyond the standard model. Therefore, the detection of DM signals is of great interest to both the particle physics and astrophysics communities.

A popular DM candidate is the so-called weakly interacting massive particle (WIMP). The future observations of HERD will be able to detect the possible DM signals in high energy CR electrons/positrons and photons.

In 2008, the PAMELA collaboration reported a CR positron excess above $\sim 10 \mathrm{GeV}$ compared with the ordinary expectation of the CR physics [1] . The AMS-02 experiment at the international space station (ISS), has reported the measurements of CR electrons, positrons [2], protons, anti-protons, 
and other nuclei. The AMS-02 results confirmed the existence of the CR positron excess observed by PAMELA and other experiments. However, the origin of these high energy positrons remains debatable. Since the positron spectrum measured by AMS-02 up to several hundred GeV does not show the cut-off feature, more detailed information of the $\mathrm{CR}$ positron spectrum at the $\mathrm{TeV}$ scale will be crucial.

In order to distinguish the origin of the high energy electrons/positrons, either from DM annihilation or from astrophysical sources, the observed electron-positron spectrum at higher precision and higher energy scales is very important. The Chinese satellite experiment DAMPE, which is launched in 2015, can precisely measure the electron-positron spectrum up to $10 \mathrm{TeV}$. In 2017 [3], the DAMPE collaboration reported their first result of CR electrons/positrons, which shows some interesting spectral features at $\sim \mathrm{TeV}$.

Compared with DAMPE, the HERD experiment has a larger acceptance and can measure the electron-positron spectrum at higher precision. Therefore, the future HERD measurement of CR electrons/positrons will be important for the high energy astrophysics and DM studies.

The PAMELA and AMS-02 data can be explained by both the astrophysical and DM sources. The best way to distinguish the two kinds of shape of cutoff is to measure the positron spectrum up to higher energy at AMS-02. However, it seems not easy to observed such difference by AMS-02 due to the limited event number at high energies. Another way to observe such differnence is to measure the fine structure at the electron plus positron spectrum by a high precision instrument, like HERD. In Fig. 1 it is shown the HERD possibility to distinguish the electron plus positron spectra in the above two scenarios. As shown in the figure it is clear that the two spectra can by clearly distinguished by 1 year data accumulation at HERD. Once the 'kink' can be identified as the positron spectrum cutoff the nature of DM particles can be well determined.

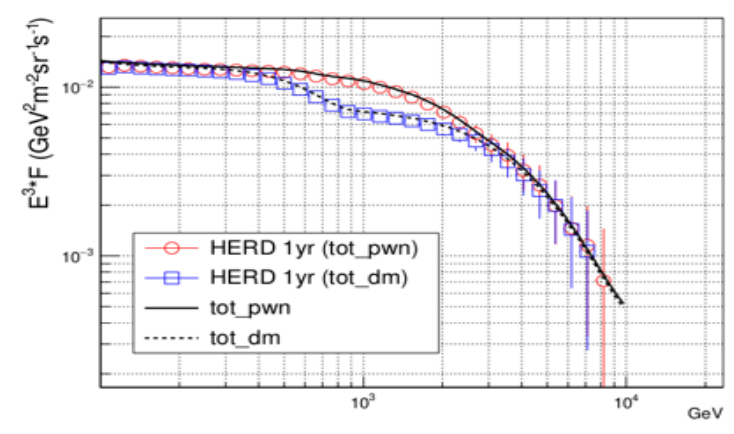

Figure 1: Simulation of HERD possibility to distinguish the total electron plus positron spectra in the two different scenarios. For 1 year data accumulation HERD can give a clear distingction for a smooth spectrum or a spectrum with a 'kink'.

\subsection{Protons and nuclei}

The unique design of HERD together with the unprecedented depth of the calorimeter and the high resolution tracker, will allow the extension of high precision measurements on proton and nuclei spectra up to the highest energies, even beyond $1 \mathrm{PeV}$. Moreover a clear identification of each nuclear species will be possible through the plastic scintillator detector, the analog readout of the silicon-tungsten tracker and by the calorimeter itself. Energy resolution for the electromagnetic 
and hadronic showers will be at the $1 \%$ and $30 \%$ level, respectively. A detailed study of the high energy photon flux and indirect signal of dark matter will also be possible. HERD will be capable of studying features in the spectra of various nuclei, like single element spectral indices and spectral hardenings/steepenings from hundreds $\mathrm{GeV} /$ nucleon up to hundreds $\mathrm{TeV} /$ nucleon. In particular the proton and helium component could be measured up to $\mathrm{PeV}$ energies, thus giving the possibility for the first direct evidence of the knee of the light component due to the reach of maximum energy provided by the source. This result would be fundamental for understanding Galactic CR acceleration/propagation processes.

Fig.2 illustrates the HERD measurements of the individual proton and Helium spectrum with a 5-yr exposure, based on a fit to the AMS-02 and ARGO-YBJ data. The HERD data can clearly reveal the knees of both protons and Helium nuclei, and can critically address the Z-dependence, A-dependence, or constant knee of different compositions, which are very important to understand the physical nature of the knee of CRs.
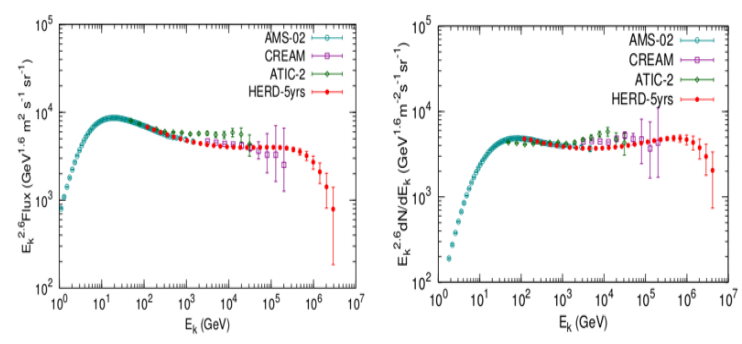

Figure 2: Expected energy spectra of protons (left) and Helium nuclei (right) measured with 5yr exposure of HERD, compared with that observed by AMS02 [4], [5], CREAM[6], and ATIC2 [7].

\subsection{Gamma-ray observatory}

Processes in the interstellar medium (ISM) contribute nearly $80 \%$ of the high-energy luminosity of the Galaxy. Although the Fermi-LAT detector has been surveying the sky for nearly 10 years with an unprecedented sensitivity, the origin of a third of the gamma-ray sources in the current Fermi LAT sources catalog (3FGL) remains unknown. HERD, with its larger effective area and better energy resolution, is expected to contribute to an improved gamma-ray diffuse emission model and to identify some of the Fermi unassociated gamma-ray sources.

Several Super Nova Remnants (SNRs) observed in the LAT energy range don't have yet a measured index in the $\mathrm{TeV}$ band. HERD in conjunction with the forthcoming H.E.S.S. galactic plane survey (and future CTA observations) will increase the number of SNRs with measured TeV index. The actual limited dataset suggest a curvature in the spectra between $\mathrm{GeV}$ and $\mathrm{TeV}$ energies, potentially reflecting a change in spectral slope of the underlying particle population's indexes. HERD will be able to observe this crucial energy range in which this curvature is observed for several SNRs, both young and middle-aged, better constraining the emitting particle population spectra.

Several fundamental questions should be answered to understand the structure of the Universe at very large scale and its history. These questions are often related to fundamental physics like the possible extension of the standard model of particle physics, quantum gravity, and the fate of 
antimatter. Gamma-ray observations in the energy band covered by HERD are required to probe several of these questions.

One of the major scientific objectives of a gamma-ray telescope is to identify the possible particle nature of dark matter (DM) by means of the production of secondary gamma-rays after the annihilation (or decay) of the DM particle candidates. The Galactic Center(GC) is expected to be the brightest source of DM annihilations in the gamma-ray sky by several orders of magnitude.Although several astrophysical processes at work in the crowded GC region make it extremely difficult to disentangle the DM signal from conventional emissions, the DM-induced gamma-ray emission is expected to be so large there that its search is of crucial relevance.The spectrum and spatial distribution of the $\mathrm{GeV}$ excess is consistent with what can be expected fromWeakly Interacting Massive Particle (WIMP) with mass of a few tens of $\mathrm{GeV}$ of annihilating to standard model particles in the inner Galactic halo. With significantly improved sensitivity, HERD could detect and separate additional gamma-ray sources in this region and thus help clarifying the nature of this excess.

The observations in the energy range above $1 \mathrm{GeV}$ by HERD will play a unique and complementary role in multi-wavelength studies across the electromagnetic spectrum with other space and ground telescopes involving radio, optical, X-ray, gamma-ray, and neutrino telescopes. The synergy among HERD, CTA and LHAASO will allow simultaneous coverage of the same sources from few $\mathrm{GeV}$ to $1 \mathrm{PeV}$ with substantial overlap of measured spectra Fig.3. Complementary observations will also contribute notably to transient science, which is crucial to study for the properties of jets, and can shed light on determining the extragalactic background light, intergalactic magnetic fields, and the validity of the Lorentz invariance.

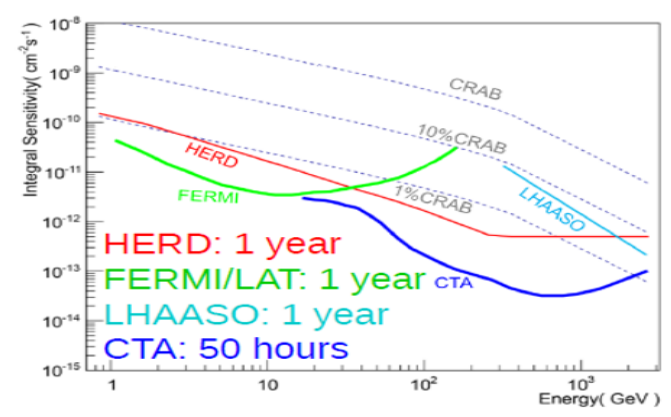

Figure 3: Comparison between Herd $5 \sigma$ senstivity and Fermi, CTA and LHAASO $5 \sigma$ sensitivity for a gamma-ray survey.

\section{The detector}

Previous space calorimeters were all built with $x-y$ orthogonal scintillator bars. The charge measurement detector, veto detector, TOF detector, and/or magnet were obliged to be installed on top of the calorimeter. So the calorimeters are only sensitive to the front. To increase significantly the geometric factor of one unique high energy radiation detection experiment, a calorimeter composed of thousands of small cubic crystals is proposed instead of the traditional design of calorime- 
ter with very long telescope "tube". Besides, better particle discrimination can be achieved by using 3-D imaging of the particle shower.

In the baseline design, HERD is composed of a 3-D cubic imaging calorimeter (CALO) surrounded by micro-strip silicon trackers (STKs) from five sides except the bottom. Then the CALO and STK are covered by the plastic scintillator detector (PSD) from outside. A Transition Radiation Detector (TRD) is located on the lateral side (Fig.4). CALO is for energy reconstruction and e/p discrimination; STK is mainly for particle tracking; PSD is for trigger of LE gamma and charge measurement; TRD is for calibration of $\mathrm{TeV}$ nuclei.

The biggest advantage over previous missions is that HERD has an effective geometrical factor of $>3 m^{2} s r$ for electrons and gamma-rays and $>2 m^{2} s r$ for protons and other nuclei, which is more than one order of magnitude higher than others. The energy resolution is about $1 \%$ for electrons and gamma-rays beyond $200 \mathrm{GeV}$, and $20 \%$ for protons from $100 \mathrm{GeV}$ to $\mathrm{PeV}$ which guarantees the best direct observation of CR spectra around "knee".

The HERD calorimeter is aimed at high resolution energy measurement of cosmic radiation with large acceptance. The calorimeter comprises three sub-systems: crystal array, IsCMOS camera and trigger sub-system. The crystal array is responsible for detection of high energy particles by recording the shower energy of incident particles. The trigger sub-system is designed to provide common trigger signals to other instruments and to make a complementary energy measurement of particles.

The Silicon TracKer (STK) is designed to perform precise particle tracks reconstruction and particle charge measurement up to $Z=26$. The HERD STK instrument is composed of five sided sub-STKs (one Top STK and four lateral STKs) surrounding the CALO. The FOV coverage should be higher than $80 \%$ to have direction of most particles measured.

The purposes of the PSD are the real-time identification of low energy gamma rays and the charge measurement of CRs with $\mathrm{Z}$ from 1 to 26. The main constraints are a high detection efficiency, a large dynamic range and a good energy resolution. Electrons and protons can easily be separated by the characterization of shower profiles in CALO, but electrons and gamma rays, both generating electromagnetic showers, can only be effectively distinguished by using a thin and light material, such as a plastic scintillator.

Ground calibration of space calorimeters can only go to $400 \mathrm{GeV}$ at maximum by using CERN SPS beam. For TeV protons, only few particles have showers all contained in the calorimeter and only visible deposited energy is recorded by detectors. Energy of primary proton is then derived by data extrapolation of low energy protons, which is not reliable. A Transition Radiation Detector (TRD) could be used to calibrate the incident particle with a dedicated energy at which transition radiation is generated but not saturated. By detecting the $\mathrm{X}$ rays generated by TR, the absolute energy of the incident charged particle in the $\mathrm{TeV}$ region can be obtained by measuring the Lorentz factor.

\section{Conclusion}

The High Energy cosmic-Radiation Detection facility is a China-led international space mission that will start its operation around 2026 on board the future China's Space Station. Thanks to its novel design, based on a 3D, homogeneous, isotropic and finely-segmented calorimeter, HERD 


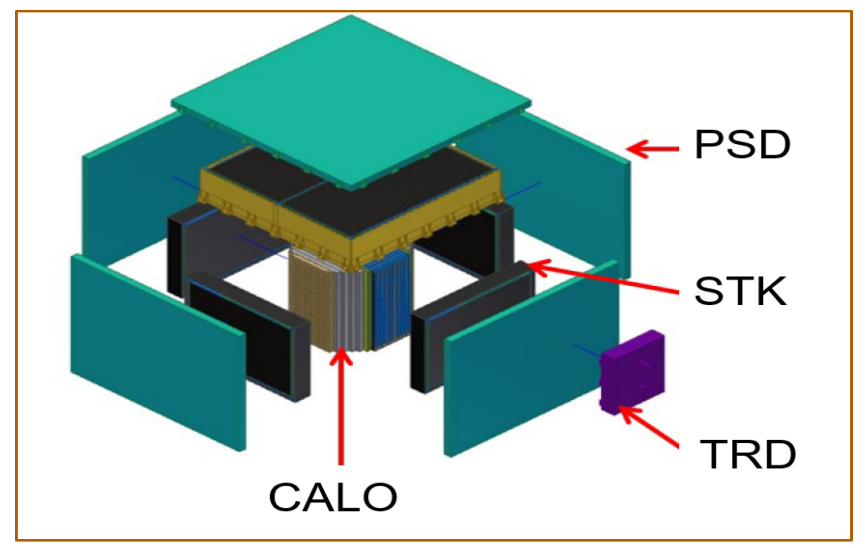

Figure 4: The Herd detector.

is expected to accomplish important and frontier goals relative to DM search, CR observations and Gamma-Ray astronomy: It will extend the measurement of $e^{+}+e^{-}$flux up to several tens of $\mathrm{TeV}$ testing the hypothesis of the expected cutoff at high energy and helping in distinguish between DM or astrophysical origin of positron excess

It will extend the measurement of $\mathrm{p}$ and $\mathrm{He}$ flux up to a few PeV testing the theory of the knee structure as due to acceleration limit

Thanks to its large acceptance and high sensitivity to gamma up to several tens of $\mathrm{TeV}$ it will search $\mathrm{g}$ for gamma-line associated to DM annihilation and accomplish a gamma-sky survey up to very high energy.

\section{References}

[1] O. Adriani et al. Observation of an anomalous positron abundance in the cosmic radiation. Nature, 458:607-609, 2009.

[2] M. Aguilar et al. First Result from the Alpha Magnetic Spectrometer on the International Space Station: Precision Measurement of the Positron Fraction in Primary Cosmic Rays of 0.5-350 GeV. Phys. Rev. Lett. 110, 2013.

[3] G. Ambrosi et al. Direct detection of a break in the teraelectronvolt cosmic-ray spectrum of electrons and positrons. Nature, 552:63-66, 2017.

[4] M. Aguilar et al. Precision Measurement of the Proton Flux in Primary Cosmic Rays from Rigidity 1 GV to $1.8 \mathrm{TV}$ with the Alpha Magnetic Spectrometer on the International Space Station. Phys. Rev. Lett., 114:171103, 2015.

[5] M. Aguilar et al. Precision Measurement of the Helium Flux in Primary Cosmic Rays of Rigidities 1.9 GV to 3 TV with the Alpha Magnetic Spectrometer on the International Space Station. Phys. Rev. Lett., 115(21):211101, 2015.

[6] H. S. Ahn et al. Discrepant hardening observed in cosmic-ray elemental spectra. Astrophys. J., 714:L89-L93, 2010.

[7] A. D. Panov et al. Energy Spectra of Abundant Nuclei of Primary Cosmic Rays from the Data of ATIC-2 Experiment: Final Results. Bull. Russ. Acad. Sci. Phys., 73:564-567, 2009. 